\title{
Semiconductor Superlattices: Artificial Crystals with Unique Electronic and Transport Properties
}

\author{
H. T. Grahn \\ Paul-Drude-Institut für Festkörperelektronik, Hausvogteiplatz 5-7, 10117 Berlin, Germany
}

Received on April 22, 2001

\begin{abstract}
Semiconductor superlattices are artificial crystals with a periodicity much larger than the lattice constants of the constituent materials. Applying an electric field parallel to the superlattice axis, i.e., perpendicular to the layers, results in unique electronic and transport properties such as miniband transport and Wannier-Stark localization, which are the basis for the observation of Bloch oscillations. At larger field strengths, resonant tunneling between different mini- or subbands dominates the electronic transport properties. Miniband transport and resonant tunneling are highly nonlinear processes resulting in interesting phenomena of self-organization of the field distribution.
\end{abstract}

Semiconductor superlattices (SLs) were proposed about 30 years ago by Esaki and Tsu [1]. In the simplest case, they consist of a periodic arrangement of two semiconductors $\mathrm{A}$ and $\mathrm{B}$ with different energy gaps with a superperiodicity $d$, which is much larger than the lattice constants $a_{\mathrm{A}}$ and $a_{\mathrm{B}}$. Due to the formation of minibands, these artificial crystals are expected to exhibit unusual transport properties such as negative differential resistance (NDR) and Bloch oscillations. It took 18 years to demonstrate that an applied electric field parallel to the superperiodicity destroys the miniband structure and results in discrete states, so-called Wannier-Stark states [2,3]. Bloch oscillations were discovered in the early 1990s [4], but so far they have only been observed in optically excited SLs. At even larger electric fields, sequential resonant tunneling (SRT) between subbands in adjacent wells will dominate the transport characteristic [5] and carriers will populate a higher subband by injection [6]. Due to the strong non-linearity of the current-voltage characteristic for miniband transport and SRT, self-sustained current oscillations $[7,8]$ and chaos $[9,10]$ have been observed in such systems.

We will focus on weakly coupled SLs, for which the SL miniband width is smaller than the broadening of the two-dimensional subbands. For this system, the transport is dominated by SRT between adjacent wells, which results in a number of peaks in the drift velocity versus electric field characteristics. As an example, Fig. 1 displays the measured drift velocity as a function of the applied electric field for a 40-period, undoped SL with $21 \mathrm{~nm}$ GaAs well and $2.5 \mathrm{~nm}$ AlAs barrier width. SRT is observed for transport from $E_{1}$ in one well to $E_{i}$ in the adjacent well with $i=2,3,4,5$, and 6 . The drift velocity exhibits several regions of negative differential velocity. When a large carrier density is introduced into the SL, either by photo-excitation or by doping, static electric-field domains are formed, which consist of two regions of constant field strength, determined by the subband spacings, separated by an accumulation layer, a charge monopole. The motion of this monopole with increasing electric field gives rise to a very peculiar $I-V$ characteristic consisting of as many current branches and current jumps as there are periods in the SL. When the carrier density is reduced, the domain formation becomes unstable. The monopole can perform a recycling motion inside the SL, which covers only part of the whole number of periods. This recycling motion appears in the experiments as current self-oscillations, when the current is recorded as a function of time. As an example, Fig. 2 shows a time trace for an n-doped,

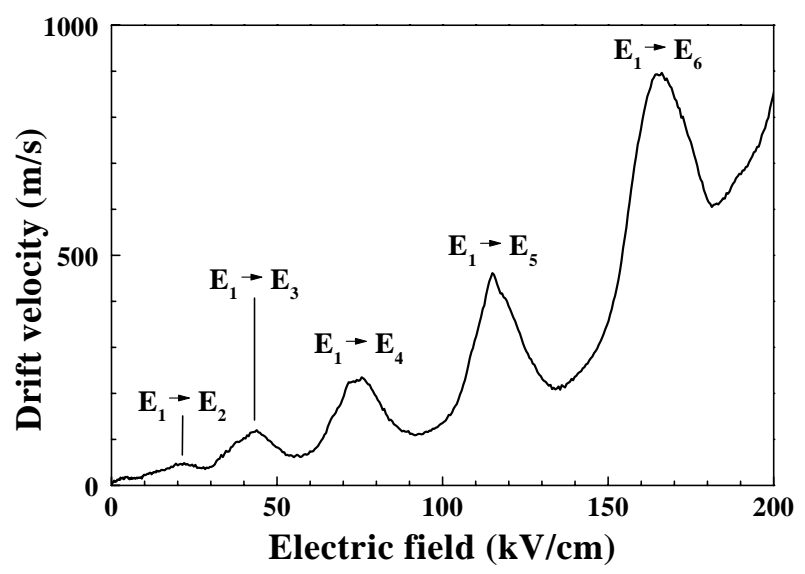

Figure 1. Drift velocity vs electric field for an undoped SL with 40 periods, $21 \mathrm{~nm}$ GaAs wells, and $2.5 \mathrm{~nm}$ AlAs barriers measured at $7.5 \mathrm{~K}$. 


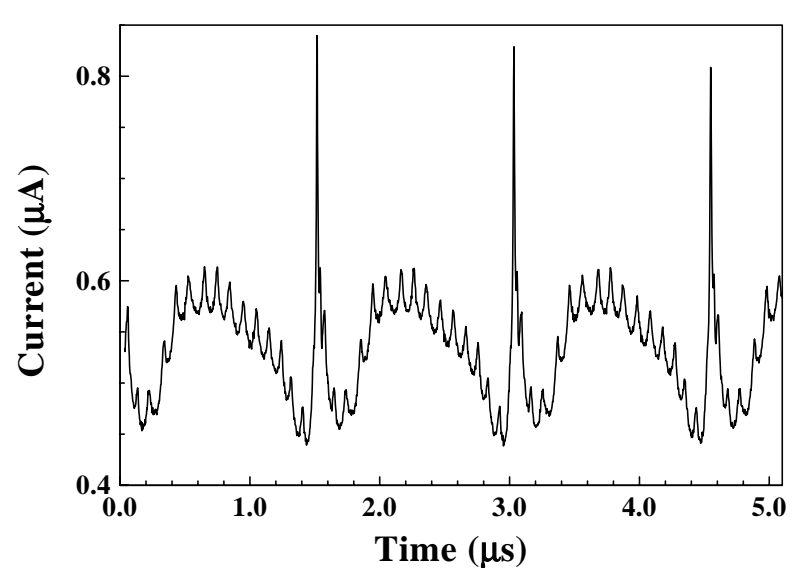

Figure 2. Spontaneous current oscillations in a doped SL with 40 periods, $9.0 \mathrm{~nm}$ GaAs wells, and $4.0 \mathrm{~nm}$ AlAs barriers for an applied voltage of $2.77 \mathrm{~V}$ and a temperature of $6 \mathrm{~K}$. There are 16 spikes in a single period of the $0.65 \mathrm{MHz}$ oscillations.

40-period SL with $9.0 \mathrm{~nm}$ GaAs wells, $4.0 \mathrm{~nm}$ AlAs barriers, and a doping density of $3 \times 10^{17} \mathrm{~cm}^{-3}$. This sample exhibits current self-oscillations with a period of $0.65 \mathrm{MHz}$. At the same time, there are 16 spikes with a frequency of $10 \mathrm{MHz}$ within a single period of the $0.65 \mathrm{MHz}$ oscillations. The spikes are due to the relocation of the charge monopole between two adjacent quantum wells. In weakly coupled SLs, the charge monopole can only reside in the wells so that the motion of the monopole from period to period becomes discontinuous. The ratio of 16 spikes divided by 40 periods demonstrates that the recycling motion of the charge monopole only covers about $1 / 3$ of the SL.

When the coupling between the wells in increased, either by reducing the barrier width or by decreasing the effective barrier height through larger electric fields, the frequency of the current oscillations increases by more than four orders of magnitude. The highest frequency observed in weakly coupled SLs is $8 \mathrm{GHz}$ [11]. Table I lists most of the investigated weakly coupled SLs in terms of the well $d_{W}$ and barrier widths $d_{B}$, the calculated subband energies $E_{i}$ and miniband widths as well as the temperature $T$ and measured frequency $f_{i}$. The increase of the frequency with decreasing barrier width and decreasing effective barrier height indicates that the frequency is mainly determined by the resonant coupling between adjacent wells. This dependence can be qualitatively seen from the WKB expression for tunneling, i.e., the tunneling probability is proportional to $\exp \left(-2 d_{B} \sqrt{2 m_{B}^{*}\left(V-E_{i}\right)} / \hbar\right)$. We therefore expect that the period (frequency) of the current oscillation is inversely (directly) proportional to the tunneling probability. A detailed analysis described in Ref. [11] shows that the observed frequencies listed in the last column of Table I can be well reproduced taking into account the current oscillations only cover about $1 / 3$ of the total number of periods of the SL.
Table I. Sample parameters and oscillation frequencies for weakly coupled SLs. $d_{W}$ denotes the well width, $d_{B}$ the barrier width, $i$ the index of the corresponding plateau of the I-V characteristic, $E_{i}$ the energy and $\Delta_{i}$ the width of the $i^{t h}$ sub- or miniband, and $f_{i}$ the measured oscillation frequency at the specified temperature $T$. The symbols are used in Fig. 3 to distinguish different samples. Solid symbols refer to weakly, open symbols to more strongly coupled SLs. Except for the $10.0 / 4.0$ sample (*), which is an (In, Ga) As / ( In, Al)As SL grown lattice matched on InP, all other SLs are GaAs/AlAs.

\begin{tabular}{ccccrrrr}
\hline $\begin{array}{c}d_{W} \\
(\mathrm{~nm})\end{array}$ & $\begin{array}{c}d_{B} \\
(\mathrm{~nm})\end{array}$ & symbol & $i$ & $\begin{array}{c}E_{i} \\
(\mathrm{meV})\end{array}$ & $\begin{array}{c}\Delta_{i} \\
(\mathrm{meV})\end{array}$ & $\begin{array}{r}T \\
(\mathrm{~K})\end{array}$ & $\begin{array}{c}f_{i} \\
(\mathrm{MHz})\end{array}$ \\
\hline 9.0 & 1.4 & $\square$ & 1 & 44.3 & 4.5 & 300 & 5,000 \\
\hline 9.0 & 1.5 & $\diamond$ & 1 & 44.5 & 3.7 & 300 & 1,500 \\
\hline 15.0 & 0.9 & $\triangle$ & 1 & 18.6 & 3.7 & 6 & 2,500 \\
\hline 10.1 & 1.4 & $\nabla$ & 1 & 36.7 & 3.4 & 300 & 1,800 \\
\hline 10.0 & 4.0 & $*$ & 1 & 48.0 & 2.0 & 6 & 1,800 \\
\hline 15.0 & 1.7 & $\bullet$ & 1 & 18.8 & 0.7 & 300 & 500 \\
& & & 2 & 75.8 & 2.8 & 300 & 8,000 \\
\hline 20.0 & 2.0 & $\bullet$ & 1 & 11.3 & 0.2 & & - \\
& & & 2 & 45.3 & 0.7 & 300 & 600 \\
& & & 4 & 102.2 & 1.7 & 300 & 1,200 \\
& & & 182.4 & 3.2 & 300 & 2,300 \\
\hline 13.3 & 2.7 & $\nabla$ & 1 & 23.3 & 0.1 & 6 & 25 \\
& & & 2 & 93.0 & 0.6 & 6 & 500 \\
& & & 3 & 211.0 & 1.6 & 6 & 1,200 \\
\hline 15.0 & 2.9 & $\boldsymbol{\Delta}$ & 1 & 19.0 & $<0.1$ & 300 & 45 \\
& & & 2 & 75.8 & 0.3 & 300 & 500 \\
& & & 3 & 171 & 0.8 & 300 & 1,700 \\
& & & 305 & 1.9 & 6 & 4,000 \\
\hline 9.0 & 4.0 & $\mathbf{\square}$ & 1 & 44.4 & $<0.1$ & 6 & 1 \\
& & & 2 & 180.0 & 0.1 & 6 & 20 \\
\hline & & & & & & \\
\hline
\end{tabular}

Figure 3 shows the measured $f_{i}$ versus calculated $f_{\text {cal }}$ oscillation frequency for the samples listed in Table I. Symbols connected with dotted lines indicate oscillations within a single sample. In this case, the increase of the frequency is solely due to a decrease of the effective barrier height by increasing the applied electric field. There is a discrepancy between the measured and calculated values of about a factor of three, which seems to be reasonable accuracy for such a simple model. Note, however, that the observed frequencies cover about four orders of magnitude (logarithmic scales for $f_{i}$ and $f_{\text {cal }}$ ). Some more strongly coupled SLs (open symbols in Table I and Fig. 3) appear to have a different scaling factor. A more detailed analysis of the current oscillation frequencies in strongly coupled SLs, which are similar 


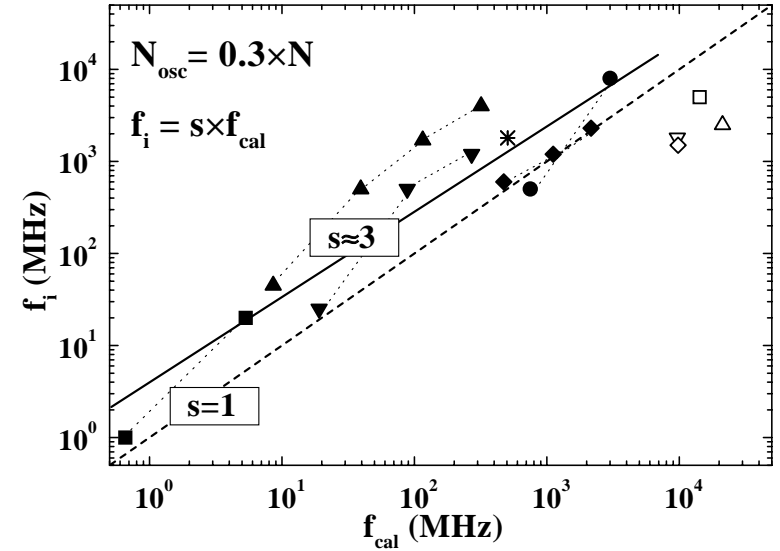

Figure 3. Measured $\left(f_{i}\right)$ versus calculated $\left(f_{c a l}\right)$ frequency for the samples listed in Table I. The same symbols connected with dotted lines indicate oscillations within a single sample in different plateaus. The dashed line indicates the condition $f_{c a l}=f_{i}$, the solid line is a least square fit to the solid data points.

to the dipole oscillations in the Gunn effect, shows that indeed strongly coupled SLs have a scaling factor of about 0.3 , when taking into account that all periods are involved in the oscillations. This value differs by a factor of about 30 from the one for weakly coupled SLs.

Finally, we present some experiments of driven current oscillations, where quasi-periodicity, frequency locking and chaos is observed. We applied a sinusoidal ac driving voltage of amplitude $V_{a c}$ with a frequency $f_{d}$ given by the golden mean $[(1+\sqrt{5}) / 2=1.618]$ times the fundamental frequency $f_{i}$ of the spontaneous current oscillations. For $V_{d c}=6.574 \mathrm{~V}, f_{d}$ was set to 49.4 MHz. At low values of $V_{a c}$, a quasi-periodic regime is observed, which ends at $37 \mathrm{mV}$. At this point, the frequency spectra begin to smear out over a limited spectral range. At $V_{a c}=40 \mathrm{mV}$, a frequency-locked state with a winding number $2 / 3$ appears, which extends up to $50 \mathrm{mV}$. For larger values of $V_{a c}$, the frequency spectra are more or less smeared out over the whole frequency range indicating the presence of chaotic oscillations. At $V_{a c}=100 \mathrm{mV}$, only a $1 / 1$ frequencylocked state remains. The actual bifurcation scenario depends strongly on the applied dc voltage. For a large dc voltage, alternating windows of quasi-periodicity and frequency-locking dominate the bifurcation diagram as a function of the amplitude of the ac driving voltage. The onset of chaos beyond $50 \mathrm{mV}$ in Fig. 4 may be related to the fact that in this regime of ac voltages the system is driven from a dynamic to a static field distribution and back.

In summary, weakly coupled SLs exhibit a nonlinear drift velocity field characteristic resulting for highly doped systems in electric-field domain formation. For intermediate carrier densities, the domain formation becomes unstable. In this case, current selfoscillations are observed with frequencies ranging from the $\mathrm{kHz}$ up to the $\mathrm{GHz}$ regime. The oscillation frequen-

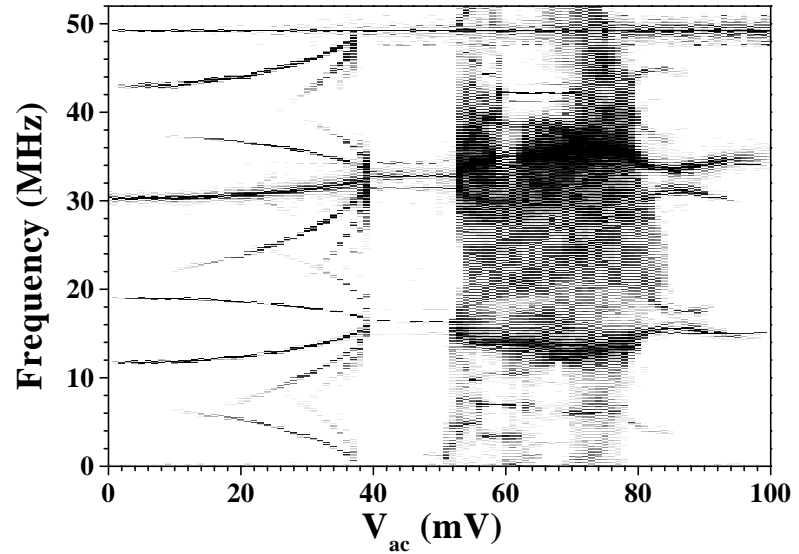

Figure 4. Power spectra of the driven current oscillations as a function of the driving voltage amplitude $V_{a c}$ for $V_{d c}=6.574 \mathrm{~V}$. The darker the area in the plot, the larger the absolute value of the amplitude of the oscillations.

cies are mainly determined by the resonant coupling between adjacent wells and the number of periods involved in the recycling motion of the charge monopole. When the SL is driven with an additional ac voltage, frequency locking, quasi-periodicity and chaotic oscillations are observed.

\section{Acknowledgments}

The author would like to thank H. Asai, L. L. Bonilla, O. Bulashenko, A. Fischer, R. Hey, J. W. Kantelhardt, J. Kastrup, K. J. Luo, M. Rogozia, K. H. Ploog, A. Wacker, and Y. Zhang for their intensive collaboration.

\section{References}

[1] L. Esaki and R. Tsu, IBM J. Res. Develop. 14, 61 (1970).

[2] E. E. Mendez, F. Agulló-Rueda, and J.M. Hong, Phys. Rev. Lett. 60, 2426 (1988).

[3] P. Voisin, J. Bleuse, C. Bouche, S. Gaillard, C. Alibert, and A. Regreny, Phys. Rev. Lett. 61, 1639 (1988).

[4] J. Feldmann et al., Phys. Rev. B 46, 7252 (1992).

[5] F. Capasso, K. Mohammed, A. Y. Cho, Appl. Phys. Lett. 48, 478 (1986).

[6] H. T. Grahn, H. Schneider, W. W. Rühle, K. v. Klitzing, and K. Ploog, Phys. Rev. Lett. 64, 2426 (1990).

[7] J. Kastrup et al., Phys. Rev. B 52, 13761 (1995).

[8] K. Hofbeck et al., Phys. Lett. A 218, 349 (1996).

[9] Y. Zhang, J. Kastrup, R. Klann, K. H. Ploog, and H. T. Grahn, Phys. Rev. Lett. 77, 3001 (1996).

[10] K. J. Luo, H. T. Grahn, K. H. Ploog, and L. L. Bonilla, Phys. Rev. Lett. 81, 1290 (1998).

[11] M. Rogozia and H. T. Grahn, Appl. Phys. A 78, in press (2001). 